\title{
Perfil do biomédico esteta e a segurança do paciente em procedimentos estéticos: uma revisão integrativa
}

\author{
Profile of the biomedical esthete and patient safety in aesthetic procedures: an integrative \\ review
}

Perfil del esteto biomédico y la seguridade del paciente em procedimientos estéticos: una revisión integradora

Adriana Pereira Trindade ${ }^{1 *}$, Murilo Tavares Amorim² ${ }^{2}$, Josane Arnaud Ferreira ${ }^{1}$, Carmen Marcele Vaz Lima $^{3}$, Beatriz Oliveira Amaro ${ }^{1}$, Jardel Fábio Lopes Ferreira ${ }^{3}$, Gustavo Moraes Holanda ${ }^{4}$, Michele Amaral da Silveira ${ }^{1}$, Gleyce de Fátima Silva Santos ${ }^{1}$, Claudia Simone Baltazar ${ }^{1}$.

\section{RESUMO}

Objetivo: Descrever por meio de uma revisão integrativa o perfil do Biomédico Esteta e a segurança do paciente em procedimentos estéticos. Métodos: Trata-se de um estudo de revisão integrativa sobre o papel do Biomédico Esteta e a segurança do paciente em procedimentos estéticos. O estudo foi realizado a partir da busca por artigos disponibilizados pelas plataformas: Pubmed, SCIELO e Portal Capes, a pesquisa priorizou textos em português e inglês e documentos das resoluções do Conselho Regional e Federal de Biomedicina. Após a seleção dos critérios de inclusão e exclusão, 7 artigos foram selecionados para análise íntegra. Resultados: Foi possível observar que relação entre os procedimentos aderidos às boas práticas de segurança do paciente e o conhecimento das técnicas pelos biomédicos, proporcionam um resultado seguro nos tratamentos estéticos e, embora ainda não existam protocolos específicos segundo a OMS para os procedimentos estéticos, nota-se que o risco de complicações e intercorrências durante ou após os procedimentos são baixos desde que haja o acompanhamento correto. Considerações finais: Diante disso, foi possível estabelecer anotável a necessidade pela contribuição com a segurança do paciente e os cuidados paliativos acercados procedimentos estéticos de modo a possibilitar uma boa resposta terapêutica.

Palavras-chave: Estética, Rejuvenescimento, Segurança do paciente.

\begin{abstract}
Objective: To describe, through an integrative review, the profile of the Biomedical Esthete and patient safety in cosmetic procedures. Methods: This is an integrative review study on the role of the Biomedical Esthete and patient safety in aesthetic procedures. The study was carried out from the search for articles made available by the platforms: Pubmed, SCIELO and Portal Capes, the research prioritized texts in Portuguese and English and documents of the resolutions of the Regional and Federal Council of Biomedicine. After selecting the inclusion and exclusion criteria, 7 articles were selected for full analysis. Results: It was possible to observe that the relationship between the procedures adhered to good patient safety practices and the knowledge of the techniques by biomedicals, provide a safe result in aesthetic treatments and, although there are still no specific protocols according to WHO for aesthetic procedures, note the risk of complications and complications during or after the procedures is low as long as there is the correct monitoring. Final considerations: In view of this, it was possible to establish the remarkable need for contribution to patient safety and palliative care about aesthetic procedures in order to enable a good therapeutic response.
\end{abstract}

Keywords: Aesthetic, Rejuvenation, Patient safety.

1 Escola Superior da Amazônia (ESAMAZ), Belém - PA *E-mail: adrianaptrindade@gmail.com

${ }^{2}$ Centro Universitário Fibra (FIBRA), Belém - PA

${ }^{3}$ Universidade Federal do Pará (UFPA), Belém - PA

${ }^{4}$ Universidade da Amazônia (UNAMA), Belém - PA

SUBMETIDO EM: 7/2020 | ACEITO EM: 8/2020 ｜ PUBLICADO EM: 10/2020 


\section{RESUMEN}

Objetivo: Describir, a través de una revisión integradora, el perfil del esteta biomédico y la seguridad del paciente en los procedimientos cosméticos. Métodos: Este es un estudio de revisión integrador sobre el papel del esteta biomédico y la seguridad del paciente en los procedimientos estéticos. El estudio se realizó a partir de la búsqueda de artículos puestos a disposición por las plataformas: Pubmed, SCIELO y Portal Capes, la investigación priorizó textos en portugués e inglés y documentos de las resoluciones del Consejo Regional y Federal de Biomedicina. Después de seleccionar los criterios de inclusión y exclusión, se seleccionaron 7 artículos para un análisis completo. Resultados: fue posible observar que la relación entre los procedimientos adheridos a las buenas prácticas de seguridad del paciente y el conocimiento de las técnicas por parte de los biomédicos, proporciona un resultado seguro en los tratamientos estéticos y, aunque todavía no hay protocolos específicos según la OMS para los procedimientos estéticos, tenga en cuenta El riesgo de complicaciones y complicaciones durante o después de los procedimientos es bajo, siempre y cuando exista la monitorización correcta. Consideraciones finales: En vista de esto, fue posible establecer la notable necesidad de contribuir a la seguridad del paciente y los cuidados paliativos sobre los procedimientos estéticos para permitir una buena respuesta terapéutica.

Palabras clave: Estética, Rejuvenecimiento, Seguridad del paciente.

\section{INTRODUÇÃO}

A estética tem alcançado cada vez mais reconhecimento e importância para a população como um todo. O profissional biomédico habilitado em estética pode realizar vários procedimentos estéticos faciais de caráter minimamente invasivos, porém os cuidados e a segurança dos pacientes devem ser priorizados mediante as possíveis complicações resultantes destes tratamentos (VIEIRA KKV e MENDES WV, 2018).

A alta procura por tais procedimentos aumenta de forma significante os erros e, consequentemente podem causar várias complicações, dos métodos adotados para a verificação dos erros nos procedimentos e a gravidade destes continuam preocupantes.

Essa preocupação com a segurança do paciente trouxe resultados positivos, onde as mudanças nas práticas diárias dos profissionais e a melhoria dos sistemas de atendimento tem modificado os cenários de cuidados (MENDES W, 2013).

O biomédico, habilitado a atuar na estética pela resolução $n^{\circ} 197$, de 21 de fevereiro de 2011 do Conselho Federal de Biomedicina (CFBM), é um profissional capacitado para reduzir de forma segura e eficaz os sinais decorrentes do envelhecimento, através de seus cursos de especialização e pós-graduação na área da estética. Além disso, esse profissional é capacitado para elaboração de tratamentos específicos através de uma anamnese minuciosa, cuidando da saúde, bem-estar e beleza das pessoas.

Segundo a resolução $n^{\circ} 241$ do Conselho Federal de Biomedicina de 29 de maio de 2014, uma das classes de procedimentos que podem ser utilizados pelo biomédico esteta no tratamento do rejuvenescimento, são os procedimentos minimamente invasivos, ou seja, procedimentos faciais invasivos não cirúrgicos como a aplicação de toxina botulínica do tipo A, preenchimentos e intradermoterapia. Pelo artigo $5^{\circ}$ dessa mesma resolução, o biomédico esteta torna-se ainda prescritor das substâncias utilizadas para tais procedimentos (CFBM, 2014).

De acordo com a Organização Mundial da Saúde (OMS) (2011), cada país deve desenvolver a sua própria estratégia para reforçar a segurança do paciente, envolvendo ações promovidas pelas instituições de saúde e ensino para reduzir a um mínimo aceitável, o risco de dano desnecessário associado ao cuidado de saúde.

No Brasil, as metas para a segurança do paciente são baseadas nas metas internacionais estabelecidas de acordo com a OMS. As ações que tratam da segurança do paciente estão sob a responsabilidade da Secretaria de Atenção à Saúde/Ministério da Saúde (SAS/MS) e são coordenadas pelo Comitê de Implementação do Programa Nacional de Segurança do Paciente (CIPNSP). 
Sabendo da importância do tema, o Ministério da Saúde (MS) editou a Portaria do Gabinete do Ministro/Ministério da Saúde (GM/MS) 529/2013, que institui o Programa Nacional de Segurança do Paciente. A Agência Nacional de Vigilância Sanitária (ANVISA) institui as ações para Segurança do Paciente, conforme Resolução de Diretoria Colegiada (RDC) no 36/2013 com objetivo de prevenir e reduzir a incidência de eventos que gerem danos ao paciente, adotando como escopo de atuação para os eventos associados à assistência à saúde, os protocolos da Organização Mundial da Saúde, que dispõe de um conjunto de metas para uma prática assistencial segura e publicados nas portarias GM/MS 1377/2013 e GM/MS 2.095/2013 (ANVISA, 2017).

Embora a segurança do paciente e a qualidade dos cuidados de saúde estejam no foco das discussões atuais, os erros decorrentes da assistência em saúde têm destaque na sociedade, pois são cada vez mais noticiados na mídia impressa, televisiva ou na internet.

Isso se explica pelos desfechos dos incidentes (sequelas graves) que causam muita repercussão. A segurança nos procedimentos estéticos é algo que deve ser levado a sério, pois, cuidar da beleza com segurança irá proporcionar a satisfação nos resultados e preservação da saúde (CARLESI KC, 2017).

Partindo do princípio de que o conhecimento do biomédico esteta contribui para ampliar os lócus de atuação nesta área da saúde, conclui-se que na biomedicina estética ainda existe uma necessidade de maior divulgação do papel do biomédico e da segurança do paciente em procedimentos estéticos faciais, principalmente por poder contribuir para as profilaxias e tratamentos nesta área de grande relevância na saúde (CRBM, 2017).

Por conta disso, o biomédico esteta tem como objetivo levar saúde aos indivíduos através da beleza, assim sendo, um meio de melhorar suas condições de bem-estar físico, utilizando-se de métodos e técnicas para tratamentos das disfunções estéticas corporais, faciais e envelhecimento fisiológico relacionados à derme e seus anexos, tecido adiposo e metabolismo, o que proporciona a prevenção de doenças, o rejuvenescimento fisiológico, melhorando a autoestima e os hábitos de vida segundo estabelecido pela Sociedade Brasileira de Biomedicina Estética (SBBME, 2018).

Por conseguinte, a biomedicina estética tem ganhado mais reconhecimento e espaço no mercado brasileiro. Deste, a aprovação da habilitação para atuar na estética pela resolução $n^{\circ} 197$, de 21 de fevereiro de 2011 do CFBM, é um profissional altamente capacitado para a realização de tratamentos de disfunções corporais e faciais decorrentes do envelhecimento (CFBM, 2011).

Entretanto, em comparação aos estudos internacionais, ainda há poucas publicações científicas brasileiras que dissertem, de maneira detalhada, os riscos de intercorrências em procedimentos estéticos minimamente invasivos, os quais podem ser feitos pelo biomédico esteta e a segurança do paciente.

À vista disso, manifestou-se o interesse dos pesquisadores na elaboração da temática, observando que é de fundamental importância para o biomédico esteta, a informação sobre a segurança do paciente. As técnicas que envolvem a região facial oferecem um risco de gerar possíveis intercorrências, no qual medidas preventivas são decisivas na conduta do profissional biomédico antes de realizar qualquer procedimento estético.

É indispensável estudos que tratem sobre a garantia, a segurança e os melhores resultados, objetivando o bem-estar e a qualidade de vida do paciente segundo as normas da ANVISA. Ao mesmo tempo contribuindo para biomédicos estetas habilitados sobre todos esses riscos e intercorrências.

Desta maneira, o presente estudo propõe discorrer por meio de uma revisão integrativa acerca de informações relevantes para entendimento dos procedimentos realizados por biomédicos e os recursos satisfatórios e consistentes que garantam a segurança do paciente nos procedimentos estéticos faciais.

\section{MÉTODOS}

Este trabalho caracteriza-se como uma revisão integrativa da literatura, distribuída em seis fases de acordo com as seguintes etapas: (1) Identificação do tema e formulação da questão de pesquisa; (2) Elaboração dos critérios de inclusão e exclusão de artigos; (3) Construção de instrumentos para coleta de dados relevantes 
dos artigos encontrados; (4) Avaliação e analise dos artigos selecionados na pesquisa; (5) Interpretação e discussão dos resultados obtidos; (6) Apresentação da revisão juntamente com referencial teórico acerca do tema em questão.

O levantamento de material foi realizado no período de fevereiro de 2019 a junho de 2020 . As bases de dados utilizadas na triagem de material, envolveram as plataformas de busca: Biblioteca Nacional de Medicina/PubMed, Scientific Electronic Library Online (SciELO) e Portal Capes.

O trabalho obteve material publicado nos anos de 2011 - 2019, tendo esse período sido escolhido em função da habilitação do biomédico em estética ser aprovada, desencadeando a era do biomédico esteta.

As palavras-chave pesquisadas foram: biomédico, biomédico esteta, procedimentos estéticos, toxina botulínica, ácido hialurônico, intradermoterapia, anatomia facial, ossos da face, pele, intercorrência em estética e segurança do paciente.

Para apuramento dos artigos, determinou-se como critérios de inclusão: artigos publicados entre 20112019, privilegiando estudos recentes acerca da temática; textos disponibilizados na integra; pesquisas científicas classificadas como originais e indexadas nas bases de dados PubMed, SCIELO e Portal Capes; e artigos disponíveis nos idiomas português, inglês e espanhol; relato de caso; revistas eletrônicas; foram incluídos documentos das resoluções do conselho regional e federal de biomedicina, documentos da ANVISA, dissertações e testes.

Por esse motivo, foram excluídos: Artigos publicados anteriormente ao ano de 2011; publicações repetidas em duas ou mais bases de dados; publicações disponíveis somente em forma de resumo; estudos em formato de carta; trabalhos voltados para a anatomia corporal e pesquisas científicas classificadas como originais, porém, que não tiveram como sujeitos de estudo o biomédico esteta e intercorrências geradas através dos procedimentos citados no trabalho.

Portanto, foram extraídos os dados de utilidade nos artigos com base em informações configuradas nas seguintes variáveis: variáveis: (i) o autor, e ano de publicação do artigo; (ii) técnicas minimamente invasivas faciais; (iii) material utilizadas em procedimentos estéticos faciais; (iv) os tipos e a frequência de intercorrências e demais incidentes; (v) segurança do paciente em procedimentos estéticos.

A procura para escolha dos títulos dos artigos foi dividida entre três pesquisadores de forma independente. Mediante aos títulos, foram designados os artigos para avaliação independente dos resumos, tendo sido encontrados 100 artigos. Os resumos dos artigos selecionados foram lidos na integra e forneceram resultados apresentados em forma de fluxograma, tabelas e quadros.

\section{RESULTADOS}

Para a elaboração do estudo foram identificados 100 artigos (Figura 1). Entre esses, 35 foram encontrados na plataforma PUBMED (busca realizada em outubro de 2019); 60 foram encontrados no SCIELO (busca realizada em outubro de 2019); e 5, encontrados no Portal Capes (busca realizada em outubro 2019).

Dentre os 100 materiais encontrados, 70 não disponibilizavam resumos e/ou não estavam nos idiomas português, inglês ou espanhol; 30 artigos são de publicações a partir do ano de 2011. Após a leitura, 70 artigos foram excluídos.

No final, foram selecionados 30 artigos para serem lidos os resumos na integra, sendo que 23 artigos estavam impróprios por apresentarem conteúdo divergente ao tema, ou por não relatarem intercorrências na área facial fora da prática do Biomédico.

Assim, foram extraídos dados de 7 estudos apresentados em forma de fluxograma, tabelas e quadros. Capítulos de livros e documentos do Conselho Regional e Federal de Biomedicina que também compõem este trabalho de revisão integrativa. 
Figura 1 - Processo de seleção dos artigos.

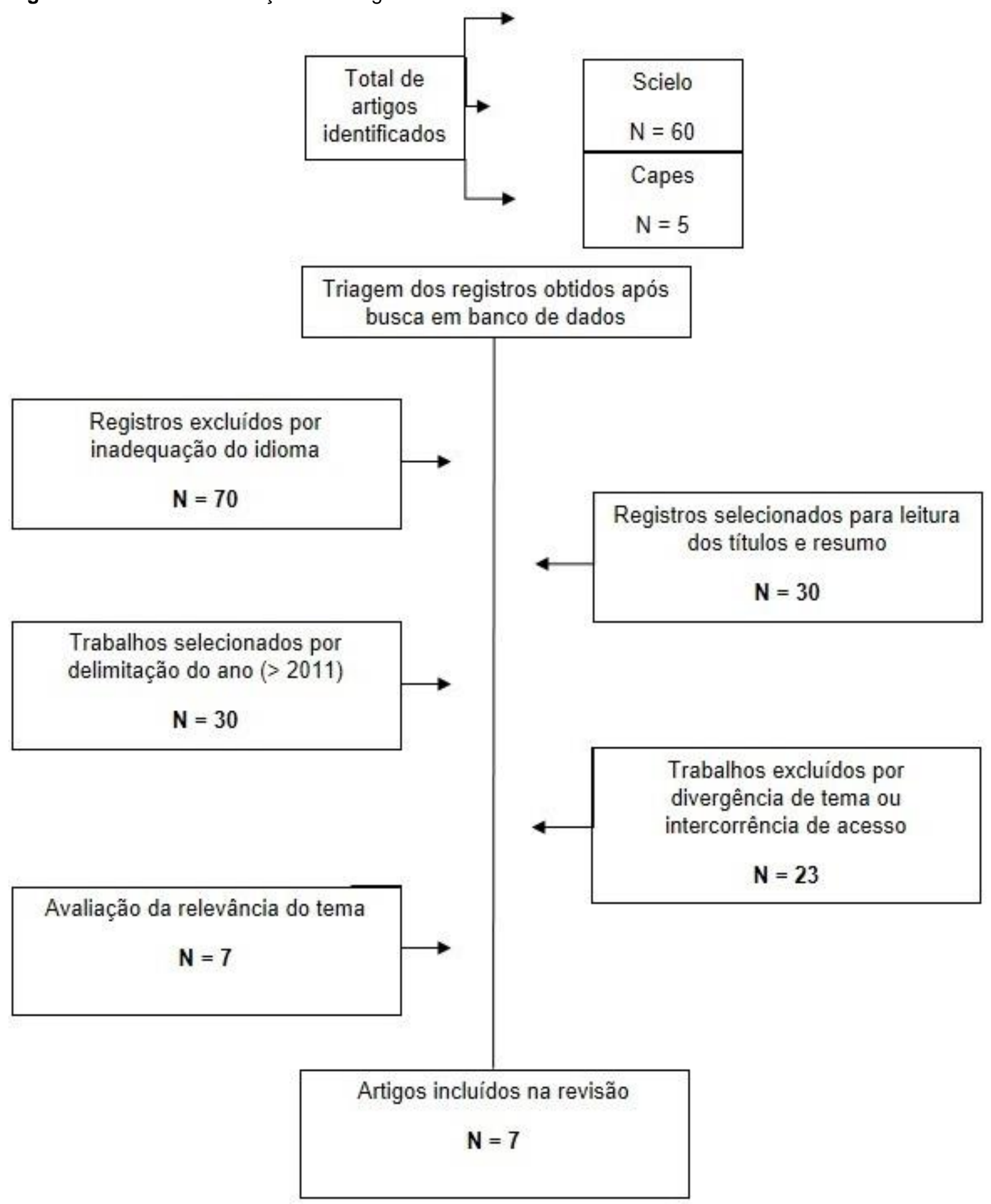

Fonte: Trindade AP, et al, 2020.

O Quadro 1 apresenta o total de artigos selecionados no estudo. Os artigos foram publicados nos anos de 2012 e 2019. Os temas centrais dos trabalhos abaixo foram procedimentos estéticos faciais como a toxina botulínica (TB), ácido hialurônico (ÁH) e a intradermoterapia, além dos cuidados paliativos com os pacientes nos procedimentos estéticos faciais. 


\section{Revista Eletrônica Acervo Saúde / Electronic Journal Collection Health | ISSN 2178-2091}

Quadro 1 - Síntese dos estudos selecionados na pesquisa.

\begin{tabular}{|c|c|c|}
\hline Autor/Ano & Resultados & Discussão \\
\hline Silva JFN (2012) & $\begin{array}{l}\text { Descrever os cuidados paliativos necessários de acordo com } \\
\text { os mecanismos de ação da toxina botulínica (TB) relevantes } \\
\text { para a compreensão do seu uso estético e aplicação em } \\
\text { procedimentos clínicos rejuvenescedores. }\end{array}$ & $\begin{array}{l}\text { A quantidade de produto a ser aplicada é analisada pelo profissional } \\
\text { Biomédico Esteta, sendo realizada de forma em que a expressão facial } \\
\text { fique natural e harmoniosa. Por ser um procedimento simples, a procura } \\
\text { está crescendo no mercado da estética, pois substitui intervenções } \\
\text { cirúrgicas. }\end{array}$ \\
\hline Nascimento CB (2016) & $\begin{array}{l}\text { Popularizar e atualizar a curiosidade que a TB desperta } \\
\text { devido aos efeitos imediatos em quase todas as vezes e } \\
\text { cuidados paliativos sobre as consequências de sua aplicação. }\end{array}$ & $\begin{array}{l}\text { A TB é contra indicada na gravidez e no aleitamento materno, pois não se } \\
\text { testaram efeitos teratogênicos e ainda não existe confirmação se a } \\
\text { substância é excretada no leite humano. }\end{array}$ \\
\hline Portella LV et al, (2016) & $\begin{array}{l}\text { Evidenciar a área da estética aplicada à biomedicina, bem } \\
\text { como esclarecer a importância do aperfeiçoamento técnico de } \\
\text { modo a contribuir para a sociedade }\end{array}$ & $\begin{array}{l}\text { O uso de preenchedores faciais está entre as principais terapêuticas } \\
\text { utilizadas pelos biomédicos estetas, a maioria é realizada de forma segura } \\
\text { e efetiva, com poucos efeitos colaterais ao paciente. }\end{array}$ \\
\hline Bratz PDE (2016) & $\begin{array}{l}\text { O estudo visa ressaltar o processo de atendimento executado } \\
\text { pelo biomédico dentro de clinicas de estéticas bem como, a } \\
\text { importância da segurança com os pacientes sobres os riscos, } \\
\text { as alternativas e os benefícios antes dos procedimentos. }\end{array}$ & $\begin{array}{l}\text { A biomedicina estética vem evoluindo muito nos últimos anos e dessa } \\
\text { forma a realização dos procedimentos, quando seguidas as pragmáticas } \\
\text { de segurança, são eficazes e seguros, com relatos de aprovação dos } \\
\text { pacientes, além de ressaltar as complicações comuns e raras nos } \\
\text { procedimentos estéticos. }\end{array}$ \\
\hline Alme & $\begin{array}{l}\text { Conhecer a aplicação do preenchimento através do ácido } \\
\text { hialurônico e o uso da hialuronidase na reversão deste } \\
\text { procedimento. }\end{array}$ & $\begin{array}{l}\text { Antes de iniciar o tratamento, os pacientes devem ser investigados } \\
\text { (Anamnese) em relação, ao histórico de distúbios hemorrágicos, herpes, } \\
\text { doenças autoimunes, gravidez, alergia, tendência a formação de } \\
\text { quelóides e uso de medicamentos. }\end{array}$ \\
\hline $\begin{array}{l}\text { Magri IO e Maio M } \\
(2016)\end{array}$ & $\begin{array}{l}\text { Abordar o AH sendo um polissacarídeo glicosaminoglicano, } \\
\text { considerado biocompátivel, pois também pode ser } \\
\text { encontrado dentro do organismo de todos os animais, mais } \\
\text { especificamente nos espaços intracelulares, onde atua com } \\
\text { função de hidratação, elasticidade e sustentação. }\end{array}$ & $\begin{array}{l}\text { A quantidade de produto a ser aplicada é analisada pelo profissional } \\
\text { Biomédico Esteta, sempre sendo realizada de forma em que a expressão } \\
\text { facial fique natural e harmoniosa. Por ser um procedimento simples, sua } \\
\text { procura está crescendo no mercado da estética, pois substitui } \\
\text { intervenções cirúrgicas. }\end{array}$ \\
\hline $\begin{array}{l}\text { Tennstedt D e } \\
\text { Lachapelle JM (2018) }\end{array}$ & $\begin{array}{l}\text { Visa determinar características benéficas do procedimento } \\
\text { intradérmico com objetivo de hidratar, revitalizar e uniformizar } \\
\text { através de sustâncias farmacológicas injetável para a face. }\end{array}$ & $\begin{array}{l}\text { A técnica apresentou como um bom aliado aos primeiros sinais de } \\
\text { envelhecimento e aporte de nutrientes com resultados duradouros, } \\
\text { havendo poucos efeitos colaterais nos pacientes. }\end{array}$ \\
\hline
\end{tabular}

Fonte: Trindade AP, et al, (2020).

REAS / EJCH | Vol.12(10) | e4783 | DOI: https://doi.org/10.25248/reas.e4783.2020 Página 6 de 9 


\section{DISCUSSÃO}

Observou-se, após análise do estudo de Silva JFN (2012) em espectro terapêutico, que o mecanismo de ação da toxina botulínica do tipo $\mathrm{A}$ em pacientes submetidos a aplicações em diversos tecidos, pode proporcionar a diminuição da amplitude do movimento, ocasionando dessa forma um relaxamento muscular prolongado, dinâmico e seguro, tendo sua funcionalidade abrangendo parâmetros preventivos em casos com formação aparente de rugas dinâmicas ou como forma de correção em rugas estáticas. Dessa forma, devido ao fato de ser um procedimento simples, sua procura está crescendo no mercado da estética, pois substitui intervenções cirúrgicas oferecendo mais segurança ao paciente.

Em uma outra perspectiva, de abordagem social, Silva JFN (2012), também refere-se ao uso dessa substância como um produto da futilidade e do consumismo, enaltecendo-a como uma das principais responsáveis pelo aperfeiçoamento e rejuvenescimento do mundo. Correlacionando as duas perspectivas estabelecidas no decorrer do estudo, nota-se então, o risco do dano estético aplicado como fator preponderante na lesão da beleza, com seu destaque na Medicina Estética, intercorrendo às consequências do risco de possíveis modificações ocorridas não desejáveis em relação ao que o paciente apresentava antes da intervenção.

Nascimento CB (2016) descreve em seu estudo, uma abordagem voltada para as complicações por meio de intervenções relacionadas ao uso da TB associado aos padrões de beleza estabelecidos na sociedade, como um fundamentador primordial dos ideais estéticos, caracterizados com a evolução social. Promove também, a busca por estudos que relacionem esses parâmetros de beleza com o uso demasiado de procedimentos estéticos em situações específicas, destacando dessa forma, a contraindicação da TB quando durante o período de amamentação, devido ao fato de não haver estudos que estabelecem se há uma conclusão se a referida droga é excretada no leite materno. Dessa forma, o autor expõe a necessidade de interromper a administração caso haja infecção no local da aplicação, seja bacteriana, fúngica ou viral como também ela deve ser evitada em clientes que forem sensíveis à mesma ou à albumina humana, orientando sempre uma boa anamnese juntamente com os critérios de segurança do paciente.

Portela LV (2016) alegou em seu trabalho os parâmetros clínicos mais aparentes, resultantes da aplicação da TB, evidenciando dessa forma, os principais efeitos resultantes perceptíveis 3 dias após a intervenção. Descrevendo seu mecanismo de ação, suas aplicações, suas contraindicações e riscos durante a realização das técnicas, o autor propõe abordagens terapêuticas que visem a manutenção de dimensão facial e o tratamento da região em aspecto geral, haja vista que o enfoque de uma nova abordagem rejuvenescedora estabelece que sejam realizados procedimentos individualmente imperceptíveis, mas que em conjunto dimensionam uma aparência tênue e natural. Nessa perspectiva, o autor infere, de forma primordial, que a busca pela beleza está associado à necessidade de auto aceitação na sociedade e a pluralidade da necessidade por mudanças, mostrando que os resultados buscados pelos clientes intercalam entre desejos individuais, as vezes inalcançáveis, efeitos colaterais imperceptíveis, necessidade pela aplicação correta por profissionais habilitados e naturalidade dos resultados pós aplicação.

Bratz PDE (2016) faz uma análise acerca dos aspectos históricos da dermatologia estética ao longo dos anos, demonstra assim, que essa área foi a principal responsável por abrir as portas para os biomédicos crescerem e serem capazes de exercer uma posição de maior destaque no mercado de trabalho atual, promovendo dessa forma, uma busca por realização profissional e melhores remunerações nesse campo. Portanto, tendo em vista essa busca demasiada e a vasta magnitude do mercado de trabalho, é normal o aparecimento de profissionais desqualificados e sem formação, exercendo funções que englobam técnicas de alto risco, tanto ao cliente, quanto ao profissional. Assim, deve ser feita a busca por locais confiáveis, com profissionais devidamente regularizados e capacitados para a realização da técnica desejada, de modo que todas as medidas preventivas durante e após a intervenção sejam realizadas e todos os resultados desejáveis sejam obtidos.

Almeida AD (2017) pressupõe acerca de complicações referentes a administração do AH, descritas na medida por meio de alterações e comprometimentos à nível sistêmico. De uma forma geral, o autor sugere a hipótese de que os preenchedores estão associados à hipersensibilidade e a reações teciduais alérgicas granulomatosas. 
Portanto, essas substâncias devem ser evitados em caso de infecção ativa em área próxima como sinusite por exemplo, processo inflamatório, imunossupressão, alergia a componentes do preenchedor ou lidocaína, gravidez ou amamentação, pois a segurança e os cuidados paliativos com o paciente são essenciais para evitar complicações durante o processo de administração dos componentes.

Magri IO e Maio M (2016), descreveram em seu trabalho que a utilização do AH pode ser realizada de forma individual ou combinada com outros materiais que preenchem e tem o mesmo papel tendo suas vantagens e modalidades aceitas. Além disso, destacam-se o uso desses materiais devido a durabilidade dos procedimentos e ao menor risco de rejeição e intercorrências.

Corroborando com o que foi supracitado, Tennstedt D e Lachapele JM (2018) dizem também que, no uso da intradermoterapia nos pacientes deve-se ter os cuidados paliativos necessários, apesar dos baixos riscos de rejeição, pois há contra indicação na gravidez, na lactação, nas doenças sistêmicas autoimunes e imunodepressão, além de ser importante evitar a exposição ao sol, assim como o uso do filtro solar deve ser mantido em caráter rigoroso antes e depois do procedimento estético.

Os estudos abordados foram capazes de demonstrar que são vários os métodos que o profissional biomédico pode utilizar na estética. Na Laserterapia podem ser usados o laser fracionado; o laser para remoção, clareamento de tatuagens e maquiagem definitiva, Lifght Emitting Diode (LED) e luz intensa pulsada. Na Eletroterapia podem ser utilizadas todas as correntes elétricas; radiofrequências; ultracavitação e ultrassom dissipado e focalizado (HIFU) (SBBME, 2019). Além dos procedimentos já citados, o biomédico esteta pode realizar avaliações e consultas estéticas, onde fazem a anamnese corporal e facial do paciente, análise das disfunções estética, podendo assim fazer a definição e a estratégia do tratamento estético a ser realizado.

O profissional em questão também é capacitado para realizar a prescrição e receita de medicamentos e substâncias que sejam para fins estéticos, que seriam: cosméticos, cosmecêuticos e nutricosméticos; medicamentos biológicos; medicamentos correlatos; medicamentos livres; medicamentos manipulados não controlados; terapias de longevidades, antienvelhecimento e envelhecimento saudável. O biomédico esteta também tem como direito e dever atuar no acompanhamento dos pacientes saudáveis durante os tratamentos estéticos, assim como supervisionar os referidos tratamentos, além de realizar treinamentos técnicos das suas equipes e colaboradores (SBBME, 2019).

A Organização Mundial da Saúde (OMS) não têm descrito atualmente, bases, protocolos ou recomendações específicas para a segurança do paciente que se submete a procedimentos estéticos. Entretanto, na portaria MS/GM no 529/2013, a OMS estabelece um conjunto de protocolos básicos para a prática assistencial segura. Nessa perspectiva, é possível inferir que esses procedimentos também possam ser empregados aos pacientes submetidos a procedimentos estéticos (BRASIL, 2014). Esses protocolos de assistência ao paciente são recomendados pela OMS tanto para situações mais comuns, como a higienização das mãos antes de qualquer procedimento, quanto para situações mais específicas, como o uso e dosagens de substâncias, cuidado com embalagens semelhantes e identificação dos pacientes.

A segurança em procedimentos estéticos é algo que deve ser ter muito cuidado. A começar pela expectativa do paciente com relação ao procedimento. Por esta razão a primeira etapa deve ser feita com uma boa anamnese e conversa para a compreensão de possíveis riscos e contraindicações, informar e tirar possíveis dúvidas sobre o procedimento e não realizar promessas de resultados inatingíveis (ANVISA, 2016).

Além disso, Os locais de trabalho para realização dos procedimentos devem respeitar as normas de espaço e higiene, os produtos devem estar armazenados em temperaturas ideais e recipientes estéreis, usar sempre materiais descartáveis e descarta-los em locais apropriados ao fim de cada procedimento, a fim de evitar possíveis contaminações.

Por fim, a formação e conhecimento necessário do profissional biomédico esteta garantirá uma maior segurança ao realizar os procedimentos minimamente invasivos proporcionando segurança e resultados positivos ao aplicá-los (Instituto Brasileiro para Excelência da Saúde /IBES, 2017). 


\section{CONSIDERAÇÕES FINAIS}

Diante dos resultados obtidos, observa-se que, embora ainda não existam protocolos específicos segundo a OMS para os procedimentos estéticos, foi possível concluir que as abordagens biomédicas, visam não somente a obtenção do rejuvenescimento da região facial com substâncias biocompatíveis, o risco de complicações e intercorrências durante ou após os procedimentos é pequeno. Considera-se que associação, entre TBA, o AH e a intradermoterapia são combinações perfeitas e representam métodos seguro passiveis de realização pelo biomédico. Acredita-se que as informações contidas neste levantamento bibliográfico sejam capazes de contribuir com a segurança do paciente e os cuidados paliativos acerca dos procedimentos estéticos que, quando bem indicados, possibilitam uma boa resposta terapêutica.

\section{REFERÊNCIAS}

1. AGÊNCIA NACIONAL DE VIGILÂNCIA SANITÁRIA. Ministério da Saúde. Manual de Gerenciamento de Resíduos de Serviços de Saúde. ANVISA; 2016.

2. AGÊNCIA NACIONAL DE VIGILÂNCIA SANITÁRIA. Implantação do Núcleo de Segurança do Paciente em Serviços de Saúde. Brasília: ANVISA; 2017.

3. ALMEIDA AD. Diagnóstico e tratamento dos eventos adversos do ácido hialurônico: recomendações de consenso do painel de especialistas da América Latina - Surg Cosmet Dermatol 2017; 9 (3): 204-13.

4. BIOMEDICINA ESTÉTICA. Campo de atuação na estética. Disponível em: https://biomedicinaestetica.com.br/campode-atuacao-na-estetica/\#.Xsw_AzpKjIU. Data da consulta: 16/ maio/ 2020

5. BIOMEDICINA. Conselho Fēeral. Resolução 241, de 29 de maio de 2014. Dispõe sobre atos do profissional biomédico com habilitação em biomedicina estética e regulamenta a prescrição por este profissional para fins estéticos. Diário Oficial, Brasília, DF, 2014. Seção 1, (129): 45-7.

6. BRASIL. Diário Oficial, O novo Código de Ética do Profissional Biomédico, 2011.

7. BRASIL. Ministério da Saúde. Documento de referência para o Programa Nacional de Segurança do Paciente. Ministério da Saúde; Fundação Oswaldo Cruz; Agência Nacional de Vigilância Sanitária. - Brasília: Ministério da Saúde, 2014. 40-9.

8. BRATZ PDE. Toxina Botulínica Tipo A: abordagens em saúde. Rev. Sau. Int, 2016, 8, 15-16.

9. CARLESI KC, et al. Ocorrência de incidentes de Segurança do Paciente e Carga de Trabalho de Enfermagem. Rev. Latinoam. Enferm. Ribeirão Preto, 2017, (25): 284.

10. CONSELHO FEDERAL DE BIOMEDICINA. Resolução n 197, de 21 de fevereiro de 2011. Dispõe sobre as atribuições do profissional Biomédico no Exercício da Saúde Estética e Atuar como Responsável Técnico de Empresa que Executam Atividades para fins Estéticos. Diário Oficial da União - Seção 1, p. 114.

11. CONSELHO FEDERAL DE BIOMEDICINA. Resolução $n^{\circ} 241$, de 29 de maio de 2014. Diário Oficial da União $n^{\circ} 129$, 9 de julho de 2014 - Seção 1, 45.

12. CONSELHO REGIONAL DE BIOMEDICINA. 1a Região - Manual Do Biomédico - Edição Digital 1 Semestre, 2017.

13. IBES. Instituto Brasileiro para Excelência em Saúde: Como evitar erros diagnósticos, 2017.

14. MAGRI IO, MAIO M. Remodelamento do terço médio da face com preenchedores. Rev. Bras. Cir. Plást., 2016; 31(4): 573-577.

15. MENDES W, et al. Características de eventos adversos evitáveis em hospitais do Rio de Janeiro. Rev. Assoc. Med. Bras., São Paulo, Out. 2013, 59(5).

16. NASCIMENTO CB. Principais complicações decorrentes do uso da toxina botulínica tipo a. Monografia de candidatura ao grau de pós-graduado em Biomedicina Estética. Monografia apresentada ao Centro de Capacitação Educacional. Recife. 2016.

17. OMS. The World Health Organization Quality of Life Assessment (WHOQOL): position paper from the World Health Organization. Social science and medicine. 2011, 41(10): 403-409.

18. OPAS. Aliança Mundial para Segurança do Paciente, 2017.

19. PORTELLA LV. Os efeitos da toxina botulínica no tratamento da espacidade: uma revisão de literatura. Rev Fisioter USP 2016; 11 (1):47-55.

20. SILVA JFN. A aplicação da Toxina Botulínica e suas complicações: Revisão Bibliográfica, Dissertação de Candidatura ao grau de. Mestre em Medicina Legal submetida ao Instituto de Ciências Biomédicas de Abel Salazar da Universidade do Porto. 2012.

21. SBBME - Sociedade Brasileira de Biomedicina Estética. A possível atuação do biomedico na estetica, 2018.

22. SBBME - Sociedade Brasileira de Biomedicina Estética. Saúde preventiva é vantajosa para o profissional, a população e para o governo - Portal do Antienvelhecimento, 2019.

23. TENNSTEDT D, LACHAPELLE JM. Effets cutanés indesirables de la mésotherapie. Ann Dermatol Venereol. 2018; 124-192.

24. VIEIRA KKV, MENDES WV. Eventos adversos e demais incidentes no cuidado estético realizado pelo biomédico. Acta Biomedica Brasiliensia, 2018; 9(1): 62-82. 